We 21 C06

\title{
Estimating a Continuous P-wave Velocity Profile with Constant Squared-slowness Gradient Models from Seismic Field Data
}

\author{
A.V. Ponomarenko* (Saint-Petersburg State University), B.M. Kashtan \\ (Saint-Petersburg State University), V.N. Troyan (Saint-Petersburg State \\ University) \& W.A. Mulder (Shell GSI BV \& Delft University of Technology)
}

\section{SUMMARY}

We inverted seismic field data for a continuous, laterally invariant P-wave velocity profile. Instead of the usual approach that involves horizontal layers with piecewise constant densities and velocities, we consider models of one or two layers with a constant gradient of the squared slowness above a homogeneous halfspace.

With a single layer above a halfspace, there are three parameters. With two layers, there are five. We solve the inverse problem by a direct grid search over a wide range of parameters. The results were compared to that of a piecewise-constant multi-layer inversion result. In the single-layer case, either the shallow or the deeper part of the model would match the multi-layer case, depending on which modes of the surface waves were selected. With two layers, a considerably better agreement is obtained over a larger depth range. Our method is limited to cases with a small $\mathrm{Vs} / \mathrm{Vp}$-ratio but has only 5 parameters. It could be a useful alternative to piecewise-constant multi-layer inversion, in particular if continuous P-velocity profiles are sought. These are sometimes better suited as a starting model for full waveform inversion than models with many discontinuities. 


\section{Introduction}

Earlier, we presented an analytical approach to invert for a continuous, smoothly varying P-wave velocity profile. We considered an acoustic model of a single layer with a linear decrease of the squared slowness with depth (constant squared-slowness gradient) bounded from above by the free surface and from below by a homogeneous acoustic halfspace. Three parameters describe the continuous velocity profile of this model. Such a medium has an analytical solution for the wavefield that can be expressed in terms of Airy functions (Brekhovskikh, 1980). This allows us to obtain a dispersion equation, similar to the multi-layer case (Socco et al., 2010). We demonstrated the feasibility of reconstructing a P-wave velocity profile from seismic data in the case of a small $V_{s} / V_{p}$-ratio by inverting the dispersion curves for a synthetic example (Ponomarenko et al., 2013b). Then, we applied the method to field data and obtained an heuristic estimate of either the shallow or the deeper part of the P-wave velocity profile, using the result of the multi-layered inversion as a reference (Ponomarenko et al., 2013a).

Here, we generalize the use of the single-layer model to estimate a P-wave velocity profile from field data by a direct grid search method over wide ranges of the parameters. Furthermore, we extend our analytic model to two layers, each with a constant gradient of the squared P-wave slowness and connected in a continuous manner. This improved the fit of the observed dispersive curves for the given field data with the inverted ones obtained after a direct grid search. The resulting velocity profile matches the reference result obtained by multi-layered inversion.

\section{Theory for forward problem}

We consider 1-D acoustic models, consisting of one or two layers with a constant squared-slowness gradient, bounded from above by the free surface and from below by a homogeneous acoustic halfspace. With one layer, the velocity follows $v_{1}(z)=v_{0} / \sqrt{1-a z}$. The deeper halfspace start at a depth $h$ and has a constant velocity $v_{2}=v_{1}(h)$, so there is no velocity contrast. We also assume that there is no density contrast. The velocity model is described by three parameters: the P-wave velocity at the surface, the depth gradient of the squared P-wave slowness and the height or thickness of this layer. The corresponding dispersion equation for the surface P-waves (Ponomarenko et al., 2013b) enables inversion of the dispersion curves for the model parameters.

The two-layer model has, again, a free-surface boundary condition at the top. The velocity in the first layer is given by $v_{1}(z)=v_{0} / \sqrt{1-a_{1} z}$ and in the second by $v_{2}(z)=v_{1}(h) / \sqrt{1-a_{2}\left(z-h_{1}\right)}$. The halfspace below starts at a depth $h_{2}$ and has a constant velocity $v_{3}=v_{2}\left(h_{2}\right)$. Again, there are no velocity contrasts. Also, we will assume there are no density contrasts, to reduce the number of parameters. Five parameters instead of three describe the model. As with one layer, we can derive the dispersion equation and invert the dispersion curves to obtain an estimate of the model parameters.

\section{Real data picking and inversion}

Earlier, we investigated the use of the single-layer model to estimate a P-wave velocity profile from pre-processed vibroseis data (Ponomarenko et al., 2013a). We focused on the dispersion curves of the P-guided waves in the seismogram in the $f, k$-domain, contained inside the yellow box of Figure 1(a). We have picked the main dispersion events of these P-guided waves as straight lines, starting from several reference points and then choosing events with a high amplitude that are close to the pre-defined positions of the straight lines. Figure 1(b) displays the picked dispersion events on top of part of the seismogram in the $f, k$-domain. There are eight curves, from the high to the low wavenumbers. They can be divided into two groups, two 'high' modes with the highest wavenumbers, five 'low' modes with the lowest wavenumbers, and one in between that we group with the high modes.

To invert for the model parameters, we adopt the misfit functional from Maraschini et al. (2010), which 


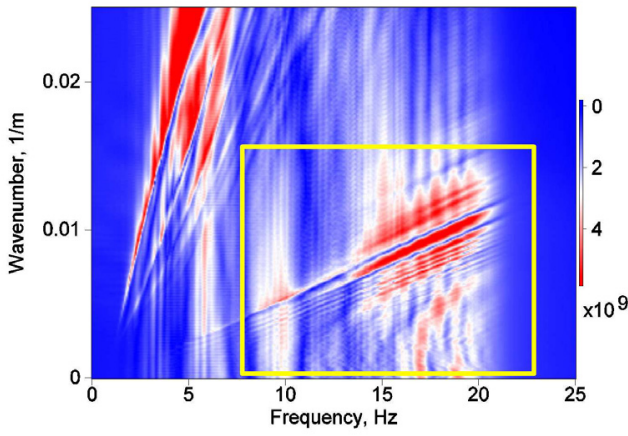

(a)

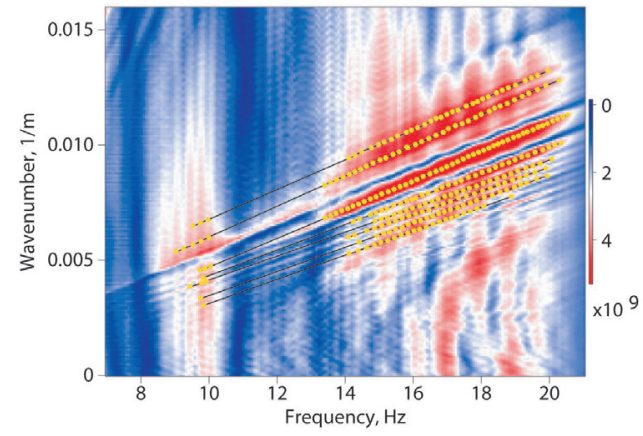

(b)

Figure 1 Real-data seismogram in the $f, k$-domain (a) and a subset (b) with the P-waves and picked dispersion events.

allows for direct minimization without requiring root searching:

$$
F_{j}(\mathbf{p})=\sqrt{\sum_{i} \mathscr{D}^{2}\left(f_{i j}, V_{i j}, \mathbf{p}\right)}
$$

Here, $\mathscr{D}\left(f_{i j}, V_{i j}, \mathbf{p}\right)$ is the left-hand side of the dispersion equation $\mathscr{D}\left(f, V_{\text {phase }}, \mathbf{p}\right)=0$, computed for frequency $f_{i j}$ and dispersion phase velocity $V_{i j}$ corresponding to a point $i$ on the dispersion curve for the picked dispersion curve or mode numbered by $j$. Note that for the single-layer model, $\mathbf{p}=\left(v_{0}, a, h\right)$, and for the two-layer model, $\mathbf{p}=\left(v_{0}, a_{1}, h_{1}, a_{2}, h_{2}\right)$. For the inversion, we minimized the functional (1) with a direct grid search over the parameter vector $\mathbf{p}$, choosing appropriate intervals and spacings after several initial tests.

\section{One layer}

We applied a 3-parameter direct grid search to both sets of picked events using the misfit functional (1). We obtained much better results if we dropped the first mode, the one with the highest wavenumbers. For the remaining high modes, we scanned the following parameter ranges: $v_{0}$ between 1.1 and $1.5 \mathrm{~km} / \mathrm{s}$, $h$ from 0.05 to $0.45 \mathrm{~km}$ and $a$ between 0.9 and $2.6 \mathrm{~km}^{-1}$. For the low modes, we considered $v_{0}$ between 1.5 and $2.1 \mathrm{~km} / \mathrm{s}, h$ from 0.3 to $1.2 \mathrm{~km}$ and $a$ between 0.2 and $1.2 \mathrm{~km}^{-1}$. The increments were chosen to be $50 \mathrm{~m} / \mathrm{s}$ for the velocity, $4 \mathrm{~m}$ for the thickness of the top layer and $0.01 \mathrm{~km}^{-1}$ for the gradient parameter. These choices were based on some initial tests. Once the global minimum on the grid was found, we started a refined search on a subset of the initial grid, with a smaller increment of $20 \mathrm{~m} / \mathrm{s}$ for the velocity but keeping the same intervals for the other parameters. The resulting parameters for the high modes are $v_{0}=1.42 \mathrm{~km} / \mathrm{s}, h=0.118 \mathrm{~km}$ and $a=2.1 \mathrm{~km}^{-1}$. For the low modes, we found $v_{0}=1.7 \mathrm{~km} / \mathrm{s}, h=$ $0.4 \mathrm{~km}$ and $a=0.9 \mathrm{~km}^{-1}$. Figure 2(a) and Figure 2(b) display 2-D cross sections through the resulting parameters of the inverse misfit functional for the high and low modes, respectively. The functional for the low modes appears to have several local minima, similar to those presented by Ponomarenko et al. (2013b). These minima show up as local maxima in Figure 2(b).

Figure 3(a) shows the predicted modal structure for the parameters estimated by using either the high or the low modes. Only the upper curves of each group are captured. Figure 3(b) displays the $V_{p}$-velocity profiles that result from the direct grid search for each of the two groups. Compared to the multi-layer inversion, the depth of the halfspace is underestimated but the velocity trend, described by $v_{0}$ and $a$, agrees better for the shallower part of the profile. This suggests that a better fit may be obtained by considering two layers above a halfspace instead of one, which we will do next. 


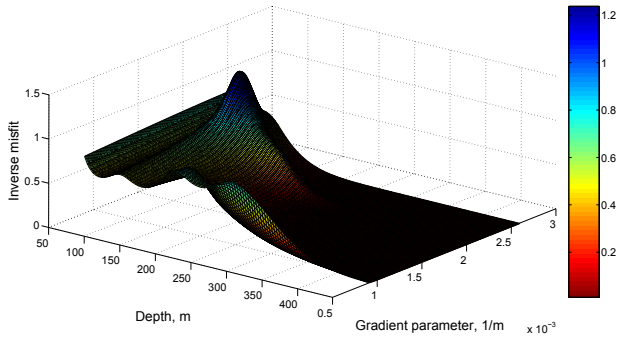

(a)

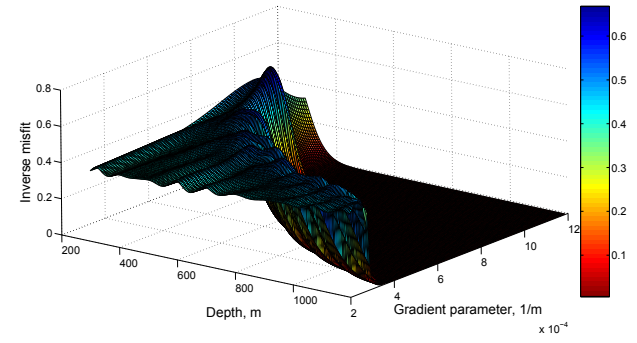

(b)

Figure 2 2-D cross sections of the inverse misfit functional for the high (a) and low modes (b) with the single-layer model. The third parameter is set to the optimal result of the direct grid search.

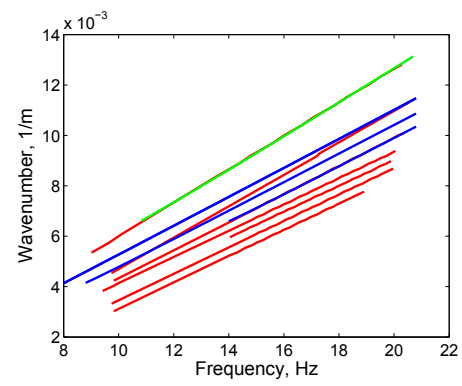

(a)

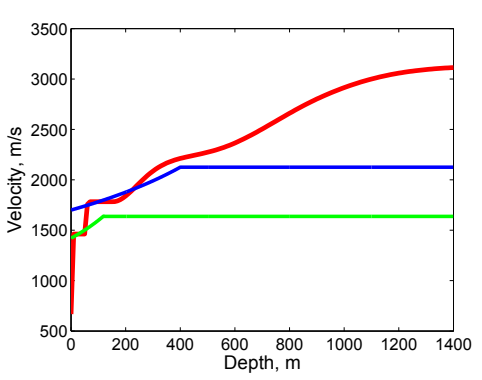

(b)

Figure 3 (a) Predicted modal structure for the high (green) and low (blue) modes together with the picked modes (red) and (b) estimated $V_{p}$ velocity profiles obtained for the high (green) and low (blue) modes, together with the multi-layer inverted profile (red) for a single-layer model

\section{Two-layered approximation}

Since the single-layer model did not give accurate results, we implemented the inversion for a twolayer model with a linear gradient of the squared slowness in the each layer, bounded from below by a homogeneous halfspace. We applied a 5-parameter direct grid search using the same misfit functional (1). First, we scanned the parameter ranges, motivated by those used for the single-layer inversion: $v_{0}$ between 1.3 and $1.6 \mathrm{~km} / \mathrm{s}, h_{1}$ from 0.05 to $0.4 \mathrm{~km}, a_{1}$ between 1.2 and $2.4 \mathrm{~km}^{-1}, h_{2}$ from 0.4 to $1.0 \mathrm{~km}$, $a_{2}$ between 0.5 and $1.0 \mathrm{~km}^{-1}$. The increments were chosen to be $20 \mathrm{~m} / \mathrm{s}$ for the velocity, $10 \mathrm{~m}$ for the thickness of the top layer, $50 \mathrm{~m}$ for the thickness of the bottom layer and $0.02 \mathrm{~km}^{-1}$ for both gradient parameters. Inversion of the high modes, excluding the first, and low modes simultaneously produced the following parameters: $v_{0}=1.42 \mathrm{~km} / \mathrm{s}, h_{1}=0.09 \mathrm{~km}, a_{1}=2.2 \mathrm{~km}^{-1}, h_{2}=0.6 \mathrm{~km}$ and $a_{2}=0.84 \mathrm{~km}^{-1}$. Figure 4(a) shows the predicted modal structure for these parameters (blue lines for the predicted, red for the picked events). The green curve in Figure 4(c) displays the corresponding $V_{p}$-velocity profile. The predicted modal structure captures only the upper curves, resulting in a large discrepancy with the multi-layer result except at smaller depths.

To capture as many curves as possible, we simultaneously inverted only the low modes inside the frequency band of 14 to $20 \mathrm{~Hz}$, where most of the picked events reside. We scanned slightly broader intervals for $v_{0}$, between 1.3 and $1.8 \mathrm{~km} / \mathrm{s}$, for $a_{1}$, between 1 and $3 \mathrm{~km}^{-1}$ and for $a_{2}$, between 0.5 and $1.5 \mathrm{~km}^{-1}$. This led to $v_{0}=1.7 \mathrm{~km} / \mathrm{s}, h_{1}=0.06 \mathrm{~km}, a_{1}=2.2 \mathrm{~km}^{-1}, h_{2}=0.9 \mathrm{~km}$ and $a_{2}=0.68 \mathrm{~km}^{-1}$. The corresponding velocity profile is shown at Figure 4(c) as a magenta curve. The predicted modal structure here captures the low modes and the resulting velocity agrees with most of the multi-layer profile with a slight underestimation of the depth of the halfspace. The shallow part of the profile again is different, which suggest we should search for the lower velocities and larger values of gradient parameter in the first layer, to obtain a more pronounced two-part velocity profile. We therefore modified the parameter ranges. especiallv for $v_{n}$ and $a_{1}$. and took $v_{n}$ between 1.1 and $1.4 \mathrm{~km} / \mathrm{s}$. $h_{1}$ from 0.05 to 
$0.4 \mathrm{~km}, a_{1}$ between 5 and $8 \mathrm{~km}^{-1}, h_{2}$ from 0.5 to $1.2 \mathrm{~km}$ and $a_{2}$ between 0.5 and $1 \mathrm{~km}^{-1}$. We used the same increments as before and only changed the one for the first gradient parameter, $a_{1}$, to $0.1 \mathrm{~km}^{-1}$. This produced $v_{0}=1.3 \mathrm{~km} / \mathrm{s}, h_{1}=0.08 \mathrm{~km}, a_{1}=6.3 \mathrm{~km}^{-1}, h_{2}=1.0 \mathrm{~km}$ and $a_{2}=0.7 \mathrm{~km}^{-1}$. Figure $4(\mathrm{~b})$ shows the predicted modal structure for the estimated parameters and blue curve at Figure 4(c) depicts the $V_{p}$-velocity profile, together with the multi-layer inversion result. The predicted modal structure again captures the low modes, but the smaller velocity value together with the larger value of the first gradient leads to a better agreement with a larger part of the multi-layer profile.

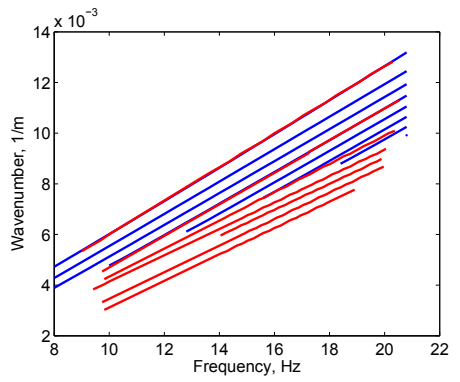

(a)

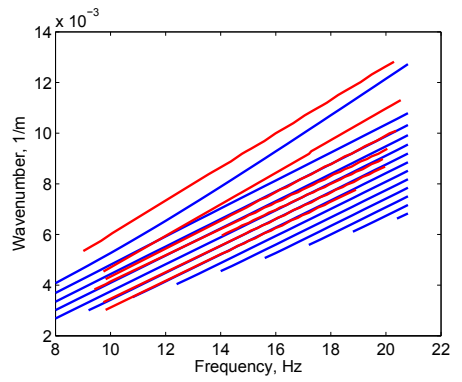

(b)

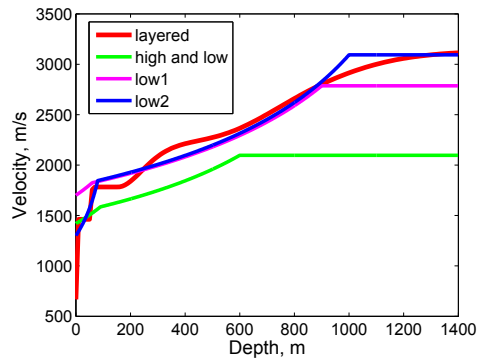

(c)

Figure 4 (a) Predicted modal structure for a two-layer model as obtained for (a) the high and low modes and (b) for only the low modes with the refined parameter ranges. (c) The corresponding estimated $V_{p}$ velocity profiles together with the multi-layer inverted profile.

\section{Conclusions}

We implemented an inversion scheme for picked P-wave dispersive events in a real shot gather using a direct grid search for one- and two-layered squared-slowness gradient profiles. We found that the singlelayer profile was not able to properly explain the dispersion curves of the field data set. In contrast, the two-layered model could better explain the observed low-wavenumber group of dispersion curves and led to a reasonable estimate of the velocity profile, compared to the result of a multi-layer inversion. Our estimate is a continuous velocity profile without a lot of detail and needs only 5 parameters to explain the multi-modal structure of the data. We conclude that the proposed method can be useful for field applications as an alternative for estimating the -wave velocity profile of acoustic and elastic data in regions with a small $V_{s} / V_{p}$-ratio near the surface.

\section{Acknowledgements}

This work was supported by Shell Global Solutions International BV under CRDF grant RUG1-30020ST-11. Additional support was provided by Saint-Petersburg State University under research grant 11.38.217.2014. The Computer Centre of SPbU provided computational resources.

\section{References}

Brekhovskikh, L. [1980] Waves in layered media. Applied mathematics and mechanics, Academic Press, ISBN 9780121305604.

Maraschini, M., Ernst, F., Foti, S. and Socco, L. [2010] A new misfit function for multimodal inversion of surface waves. Geophysics, 75(4), G31-43, doi:10.1190/1.3436539.

Ponomarenko, A.V., Kashtan, B.M., Troyan, V.N. and Mulder, W.A. [2013a] Estimation of a P-wave velocity profile from elastic real data based on surface wave inversion. Near Surface Geoscience 2013 - 19th European Meeting of Environmental and Engineering Geophysics, Bochum, Germany, 9-11 September 2013.

Ponomarenko, A.V., Kashtan, B.M., Troyan, V.N. and Mulder, W.A. [2013b] Surface wave inversion for a P-wave profile via estimation of the squared slowness gradient. $75^{\text {th }}$ EAGE Conference \& Exhibition, Extended Abstracts, London, UK, doi:10.3997/2214-4609.20130945.

Socco, L., Foti, S. and Boiero, D. [2010] Surface-wave analysis for building near-surface velocity models - established approaches and new perspectives. Geophysics, 75(5), 75A83-102, doi: 10.1190/1.3479491. 